\title{
Metal-insulator transitions in bilayer electron-hole systems in transition metal dichalcogenides
}

\author{
S. T. Chui $\odot,{ }^{1}$ Ning Wang $\odot,{ }^{2}$ and B. Tanatar $\oplus^{3}$ \\ ${ }^{1}$ Bartol Research Institute and Department of Physics and Astronomy, University of Delaware, Newark, Delaware 19716, USA \\ ${ }^{2}$ Department of Physics, Hong Kong University of Science and Technology, Clear Water Bay, Hong Kong \\ ${ }^{3}$ Department of Physics, Bilkent University, 06800 Ankara, Turkey
}

(Received 21 May 2021; revised 3 October 2021; accepted 16 November 2021; published 29 November 2021)

\begin{abstract}
We investigated metal-insulator transitions for double-layer two-dimensional electron-hole systems in transition metal dichalcogenides stacked on opposite sides of thin layers of boron nitride. The interparticle interaction is calculated by including the screening due to the polarization charges at different interfaces, including that at the encapsulation and at the substrate of experimental structures. We compute and compare the energies of the metallic electron-hole plasma and the proposed insulating exciton solid with fixed-node diffusion Monte Carlo simulation including the high valley degeneracy of the electron bands. We found that for some examples of current experimental structures, the transition electron/hole density is in an experimentally accessible range between $4.1 \times 10^{12} \mathrm{~cm}^{-2}$ and $14.5 \times 10^{12} \mathrm{~cm}^{-2}$ for spacer thicknesses between 2.5 and $7.5 \mathrm{~nm}$. Our result raises the possibility of exploiting this effect for logic device applications.
\end{abstract}

DOI: 10.1103/PhysRevB.104.195432

\section{INTRODUCTION}

There has been much recent interest in bilayer electronelectron (e-e) and electron-hole (e-h) systems in graphene and transition metal dichalcogenides (TMDCs) stacked on opposite sides of a boron nitride (BN) film of thickness $d$ [1-6]. External gate voltages are applied so that electrons and holes are on opposite sides of the BN spacer. This follows earlier interests in the physics of the two-dimensional electron gas in single and double layers in Si-MOSFET and in GaAs heterostructures [7]. An attractive feature of the graphene and the TMDC systems is the high mobility that can be achieved, thus promising practical applications.

An electron-hole system can exist as a collection of excitons or in a metallic state of an electron-hole plasma, as has been observed in bulk Ge under laser excitation [8-10]. Most previous studies consider the collection of excitons to form a fluid [11]. We recently found that in the parameter range of interest the exciton solid is more stable than the exciton fluid. This solid exhibits a supersolid behavior as an approximately quantized Coulomb-drag resistance [6]. The metal-insulator transition between the exciton solid and the electron-hole plasma is the focus of the present study. Metal-insulator transitions make possible the application of the present structure as logic devices. Graphene has also been considered for this application but because it does not have a band gap, the small but finite current makes the application difficult [12]. For practical applications, the exciton energy should be higher than room temperature. This exciton energy is inversely proportional to the Bohr radius. As an example we consider $\mathrm{WSe}_{2}$ for both the top and bottom layers. We shall express lengths in terms of the Bohr radius of $6 \AA$, corresponding to that for the electron with effective mass of $0.45 m_{e}$ [13] and a geometric mean dielectric constant discussed below of 5.1. The corresponding Rydberg (Ry), our unit of energy, is $0.235 \mathrm{eV}$. The hole mass is $0.9 m_{e}$. The exciton reduced mass is $\mu=0.31 m_{e}$ The Bohr radius for the exciton discussed later, which involves the reduced mass, is equal to $8.7 \AA$. The exciton Rydberg is $\mathrm{Ry}_{\mathrm{exc}}=0.157 \mathrm{eV}$. The exciton energy is much higher in the TMDC system [14], because its Bohr radius, $a_{B}=6 \AA$, is much smaller than that in the graphene system, $a_{B, \text { graphene }}=90 \AA[15]$, which is comparable to that in GaAs.

In this paper we included the screening of the Coulomb interaction by the polarization charge density at the interfaces of typical realistic experimental structures and found that the exciton energy can be an order of magnitude less than previous estimates that does not consider the complete structure. We extended our previous fixed-node diffusion quantum Monte Carlo calculation for the single layer electron gas $[16,17]$ to the current bilayer electron-hole system with the screened potential for different spacer thickness $d$. Previous many-body calculations $[11,18-24]$ were motivated by the GaAs heterostructures and do not correspond to the parameter range of the TMDC system. For bulk Ge, the large number of valleys lower the energy of the electron-hole fluid at high densities [8]. For the bilayer system there are two hole valleys and 6/12 electron valleys for odd/even number of layers [25]. We included this effect in our calculation and found it to be important. For $d$ between 2.5 and $7.5 \mathrm{~nm}$, we found for typical examples of experimental structures, the metal-insulator transition to occur at electron-hole densities in an experimentally accessible range between $4.1 \times 10^{12} \mathrm{~cm}^{-2}$ and $14.5 \times 10^{12} \mathrm{~cm}^{-2}$. We now describe our results in detail.

\section{SCREENING}

We consider a typical experimental structure consisting of two TMDC layers of thicknesses $2 \mathrm{~nm}$ separated by a BN layer of thickness $d$ that is of the order of $5 \mathrm{~nm}$. The structure is encapsulated by boron nitride films $\mathrm{BN}_{\text {top }}$ and $\mathrm{BN}_{\text {bottom }}$ of 


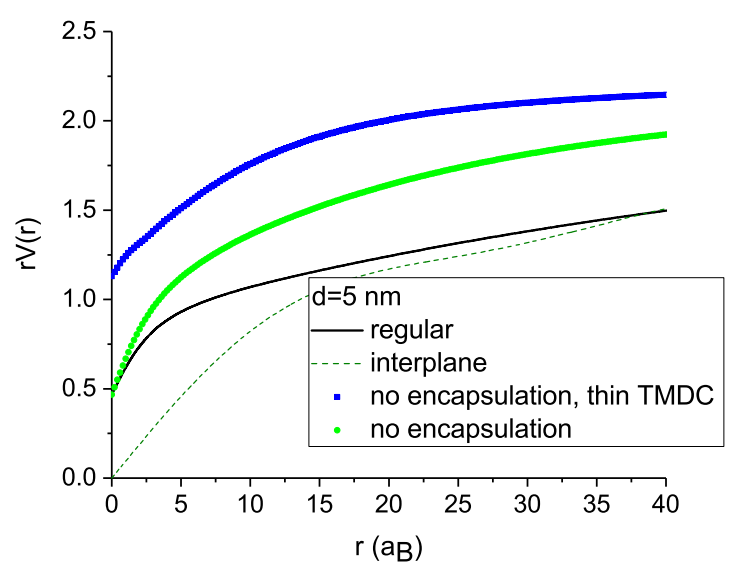

FIG. 1. The real-space intralayer potential $r V_{a}(r)$ with encapsulation (regular), with no encapsulation, no encapsulation and very thin TMDC, and results for the interlayer potential with encapsulation. $r V$ is in units of $e / \epsilon_{\mathrm{BN}}, d=5 \mathrm{~nm}$.

thicknesses 10 and $20 \mathrm{~nm}$, respectively. On one side of this structure is $\mathrm{SiO}_{2}$; on the other side, vacuum. The effect of the induced surface polarization (the "image charge" [26]) for a single two-dimensional structure was discussed by Keldysh [27] and applied to the study of excitons in single layer structures with isotropic dielectric constants [28]. Both BN and the TMDC possess hexagonal symmetry so that the dielectric constants exhibit diagonal components $\epsilon_{z}$ and $\epsilon_{x y}$ along the $z$ direction and the $x y$ plane. These dielectric constants are given [29] for $\mathrm{BN}$ as $\epsilon_{z, B N}=3.76, \epsilon_{x y, B N}=6.93$; for $\mathrm{WSe}_{2}$ as $\epsilon_{z, \mathrm{TMDC}}=7.6, \epsilon_{x y, \mathrm{TMDC}}=15.7$; and for $\mathrm{SiO}_{2}$ as 3.6. The dielectric properties are characterized by $\epsilon=\left(\epsilon_{z} \epsilon_{x y}\right)^{1 / 2}$ and the ratio $\gamma=\epsilon_{x y} / \epsilon_{z}$. The geometric means yield $\epsilon_{B N}=5.1$ and $\epsilon_{\mathrm{TMDC}}=10.9$. Furthermore, $\epsilon_{\mathrm{TMDC}} / \epsilon_{B N}=2.1, \gamma_{B N}=1.35$, and $\gamma_{\mathrm{TMDC}}=1.43$. We have previously considered the case of two thin TMDC films separated by a BN film without considering the effect of the encapsulation [6]. We extended this approach and solved the electrostatics problem of a point charge in our circularly symmetric anisotropic dielectric multilayer structure by separating the space into different regions of different dielectric constants and relate the Fourier transform of the potential $\tilde{V}(q)$ in different regions by matching the tangential (normal) components of the electric (displacement) fields at the interfaces. The general solution is then obtained with the transfer matrix method.

From $\tilde{V}(q)$ we have computed the screened potential $V(r)$ in real space. As the distance becomes larger than the multilayer thickness, the effect of screening diminishes, and $r V(r)$ approaches the limit of $e /\langle\epsilon\rangle$ with $\langle\epsilon\rangle=(1+$ $\left.\epsilon_{\mathrm{SiO}_{2}}\right) / 2$. In Fig. 1 we show the intralayer potentials $V_{a}$ in units of $e / \epsilon_{B N}$ close to the origin. For no encapsulation and thin TMDCs our previous result [6] shows that for small $r$, $r V(r)\left(\epsilon_{B N} / e\right)$ approaches $\epsilon_{B N} /\left\langle\epsilon^{\prime}\right\rangle=1.67$ with $\left\langle\epsilon^{\prime}\right\rangle=(1+$ $\left.\epsilon_{B N}\right) / 2$, close to our numerical result of 1.2 at small $r$ in this figure. In the presence of the TMDC, close to $r=0$, a very simple effective medium idea suggests that $r V(r)\left(\epsilon_{B N} / e\right)$ approaches $\epsilon_{B N} /\left\langle\epsilon^{\prime \prime}\right\rangle$ where $\left\langle\epsilon^{\prime \prime}\right\rangle=\left(\epsilon_{\mathrm{TMDC}}+\epsilon_{B N}\right) / 2$. Numerically $r V(r)\left(\epsilon_{B N} / e\right)$ approaches 0.5 in this figure, close to our estimate of $\epsilon_{B N} /\left\langle\epsilon^{\prime \prime}\right\rangle=0.64$. There is very little dependence of the intralayer potential on $d$ at small distances.

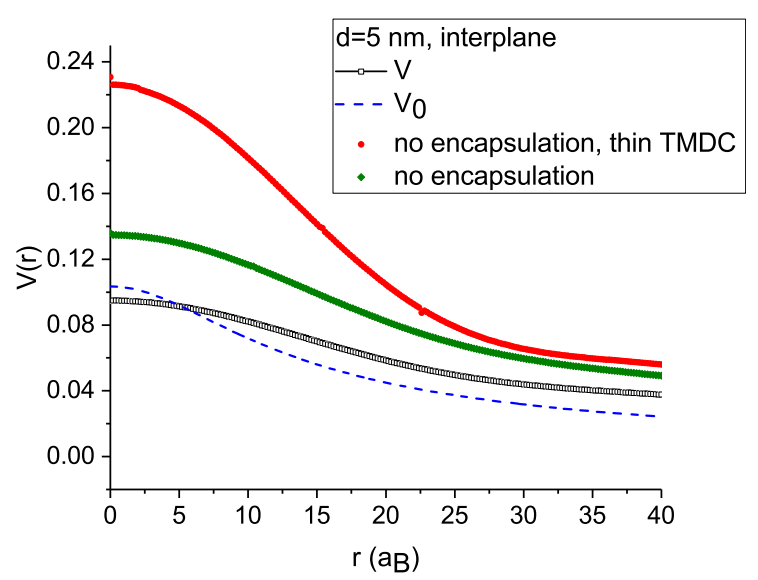

FIG. 2. The real-space interlayer potential $V_{e}(r)$ with encapsulation, with no encapsulation, no encapsulation and very thin TMDC and the bare Coulomb potential $V_{0} . V$ is in units of $e /\left(a_{B} \epsilon_{B N}\right)$ and $d=5 \mathrm{~nm}$.

We next turn our attention to the interlayer potential $V_{e}$ for the interaction between charges on opposite sides of the $\mathrm{BN}$ spacer. In Fig. 2 we show the real-space interlayer potential $V_{e}(r)$ in units of $e /\left(\epsilon_{B N} a_{B}\right)$ for $d=5 \mathrm{~nm}$ together with results for the corresponding structures with no encapsulation, no encapsulation and very thin TMDC, and, for reference, the Coulomb potential in $\mathrm{BN} V_{0}=e /\left[\left(d \gamma_{B N}+t_{\mathrm{TMDC}} \gamma_{\mathrm{TMDC}}\right)^{2}+\right.$ $\left.r^{2}\right]^{1 / 2} / \epsilon_{B N}$ in which $t_{\text {TMDC }}$ is the thickness of the TMDC film. The potential for our structure is softer than $V_{0}$ because as $r$ increases there is less screening and thus the potential decreases less rapidly. The encapsulation and the finite TMDC thickness produces a significant effect. For the case without the encapsulation and very thin TMDCs, in our previous study [6] we found that the screened potential for the interaction at small transverse distance $r$ approaches $V(r)=V_{0}(r) \epsilon_{B N} / \epsilon_{\mathrm{eff}}$, where the effective dielectric constant is given by $1 / \epsilon_{\text {eff }}=$ $\left(1-\beta^{2}\right), \beta=\left(1-\epsilon_{B N}\right) /\left(\epsilon_{B N}+1\right)$ is the well-known image charge in elementary electrostatics [26]. Now $\beta=0.672$ and $1 / \epsilon_{\mathrm{eff}}=\left(1-\beta^{2}\right)=0.548$. Our estimate thus suggests that for no encapsulation and very thin TMDC the potential at $r=0$, given by $e /\left(\epsilon_{\mathrm{eff}} \gamma d\right)$, is equal to $0.248\left(e / a_{B} / \epsilon_{B N}\right)$. This is close to the $r=0$ value of $0.23\left(e / a_{B} / \epsilon_{B N}\right)$ in this figure. In contrast to the intralayer potential, the dependence of the interlayer potential on the $\mathrm{BN}$ spacer thickness is significant. This is illustrated in Fig. 3.

The energy due to the interaction of a charge and the uniform charge backgrounds of density $\sigma=1 /\left(\pi r_{s}^{2} a_{B}^{2}\right)$ is given by

$$
E_{b g}=\left[\tilde{V}_{e}(q=0)-\tilde{V}_{a}(q=0)\right] /\left(\pi r_{s}^{2}\right) \quad(\mathrm{Ry}),
$$

where $\tilde{V}_{a}\left(\tilde{V}_{e}\right)$ is the intralayer (interlayer) interaction. If we approximate $V$ by a Coulomb potential with an effective screening constant, we obtain $E_{b g}=-\sigma d /\left(2 \epsilon_{\text {eff }}\right)$, as we expected from elementary electrostatics. Numerically, we found that $-E_{b g} /(\sigma)=6.19,10.08,15$ Ry for $d=2.5,5,7.5 \AA$, corresponding to an $\epsilon_{\text {eff }} / \epsilon_{B N}=1.1,0.9,0.8$. For the electrically non-neutral e-e systems, the corresponding background energy is inversely proportional to the first power and not the second power of $r_{s}$ and much larger [30] in magnitude. The 


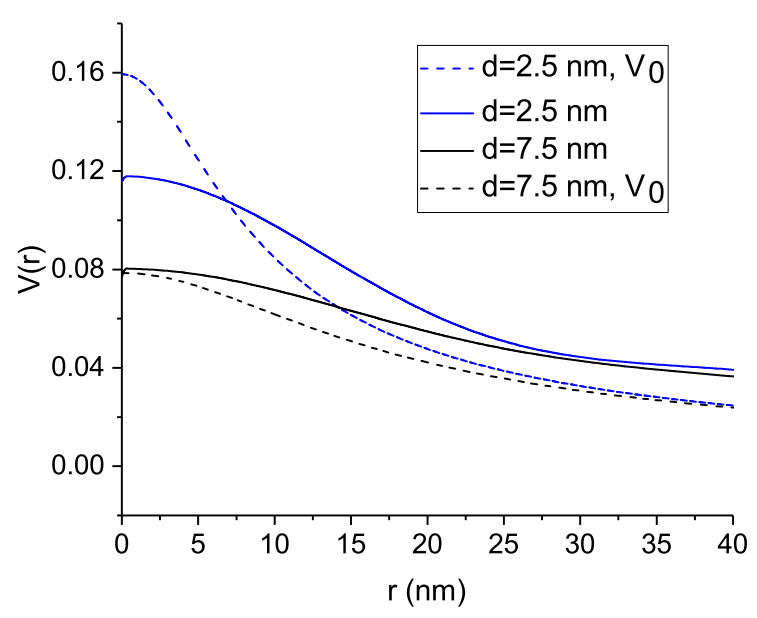

FIG. 3. The real-space interlayer potential $V(r)$ with encapsulation for two values of $d$ and the bare Coulomb potential $V_{0}$.

origin of the energy scale is shifted so that most of the energies per particle are negative for the e-e system, whereas most of the energies per particle for the present system are positive.

\section{EXCITON}

To gain some intuition on the properties of the system, we first discuss the physical property of a single exciton in the bilayer $\mathrm{WSe}_{2}$ structure with electrons and holes on opposite sides and the $\mathrm{BN}$ spacer in between. Because our potential is circularly symmetric, the exciton wave function $\chi$ can be separated into a radial and an angular component, $\chi(r, \phi)=$ $\psi(r) e^{i l \phi}$. We discretize the radial equation and solve for the eigenvalue problem of the matrix, which is tridiagonal but not symmetric, with the EISPACK routine rt.f in double precision.

The bound state eigenvalues in units of the exciton Rydberg, $R y_{\text {exc }}$, as a function of different BN thicknesses are shown in Fig. 4 for $l=0$ (lines). Also shown is an analytic estimate [6] for the ground-state energy using the second

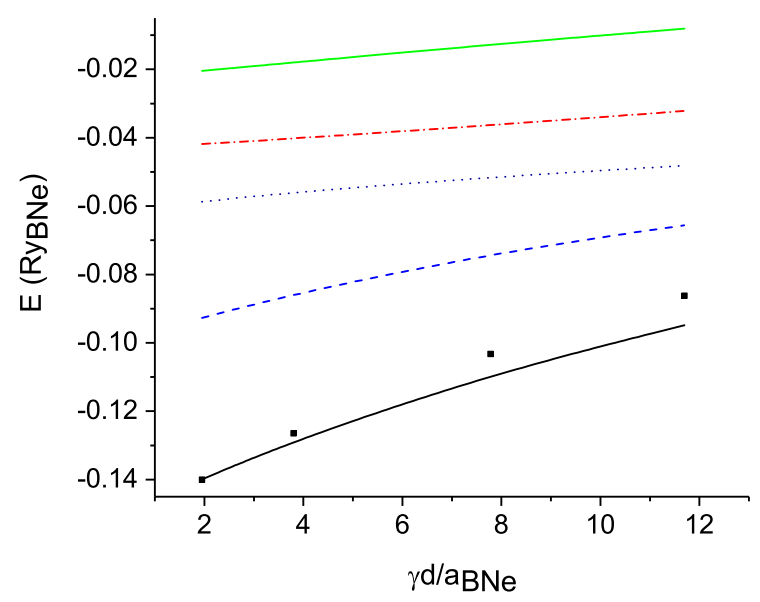

FIG. 4. Exciton binding energies in units of Ry as a function of the BN thickness normalized by the exciton Bohr radius for $l=0$. The different color curves correspond to different eigenstates. Also shown is an analytic estimate for the ground-state energy (solid black square).

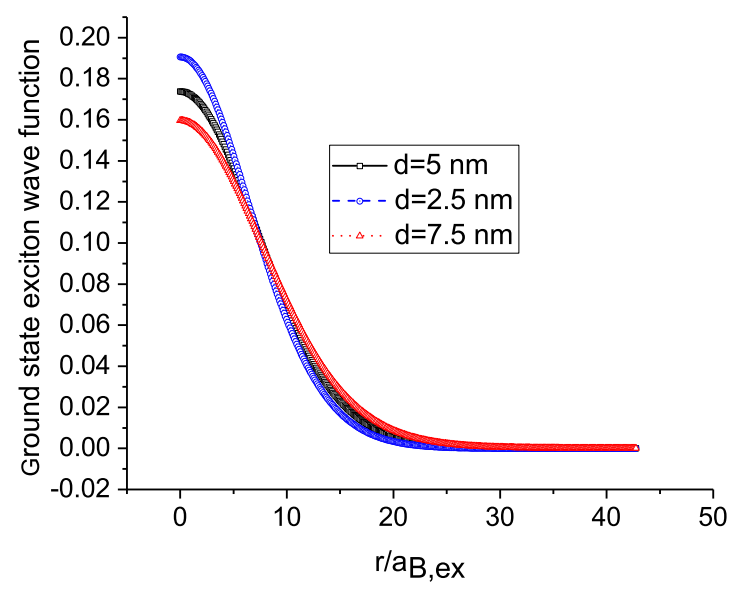

FIG. 5. Exciton wave functions for $l=0$ for different $d$ as a function of radial distance in units of the Bohr radius.

derivative of the interplane potential, which agrees well with the numerical result. Our result is an order of magnitude smaller than previous estimates that uses a potential without the effect of the encapsulation [14].

The ground-state wave functions for three different values of $d$ are shown in Fig. 5. We have previously considered the large $d$ limit for the potential $V_{0}$ and found that the width of the wave function $\xi$ is of the order of $\xi \approx d\left(a_{B} / d\right)^{1 / 4}$. The size of the wave function is larger than this estimate because our potential is softer. Also, because the potential does not change much as $d$ is decreased, the ground-state wave function has a weak dependence on $d$. The electroluminescence is proportional to the probability of finding the electron and the hole on top of each other and thus equal to $\psi_{\text {exciton }}^{2}(r=0)$. We next turn our attention to the metal-insulator transition.

\section{FIXED-NODE DIFFUSION MONTE CARLO SIMULATION}

To investigate the metal-insulator transition, we have calculated the energies of the electron-hole plasma and the hexagonal quantum exciton solid by extending our previous fixed-node diffusion Monte Carlo simulation [16,17,31]. This approach solves the many-particle Schrödinger equation in imaginary time for a finite number of particles under periodic boundary conditions. For our problem, the potential with the encapsulation is used. To enhance the speed of the computation, the solution is assumed to have the same node structure of a trial function of the form [31]

$$
\Psi=D \exp \sum_{i<j, a, b} u_{a b}\left(r_{i j}\right),
$$

where $D$ is a product of $n$ Slater determinants of singleparticle wave functions for particles in the $n$ different valleys. For the fluid (solid) phase, the single-particle wave functions are plane waves (Gaussian orbitals localized on the lattice sites.) The second factor is a Jastrow correlation factor for particles between layer $a$ and layer $b$. We next discuss the choice of $u_{a b}$.

Our interparticle interaction is given by
$\sum_{q, a b} \tilde{V}_{a b}(q) \rho_{a}(q) \rho_{b}(-q) / 2$, where $\tilde{V}_{a=b}=\tilde{V}_{a}\left(\tilde{V}_{a \neq b}=\tilde{V}_{e}\right)$ is 
the intralayer (interlayer) interaction. For the electron-electron fluid system, the simplest way is just to have

$$
u_{a b}=-1 / S+\left[1 / S^{2}+\tilde{V}_{a b}\right]^{1 / 2},
$$

where $S$ is the static structure factor for noninteracting electrons. For the electron-hole system, $u_{a b}$ becomes imaginary for $\tilde{V}_{e}<0$. Thus, this approach cannot be used. De Palo et al. [19] have investigated the phase diagram of a two spin particle hole system with two layers interacting with an isotropic Coulomb potential with fixed-node Monte Carlo simulation in a different parameter regime of $d / a_{B}<3$. We have used a modification of their $u$.

We introduce new operators that are linear combinations of charge densities $\rho_{1,2}$ on the two layers:

$$
\rho_{ \pm}=\left(\rho_{1} \pm \rho_{2}\right) / r,
$$

with the normalization factor $r=2$ as $\rho$ involves the product of two field operators. If we interpret 1 as pseudospin up and 2 as pseudospin down, then $\rho_{+}\left(\rho_{-}\right)$is the particle (pseudospin) density. The interparticle interaction can be written in diagonal form as $\left|\rho_{+}\right|^{2} \tilde{V}_{+}+\left|\rho_{-}\right|^{2} \tilde{V}_{-}$, where $\tilde{V}_{ \pm}=\left(\tilde{V}_{a} \pm\right.$ $\left.\tilde{V}_{e}\right) / 2$. This motivated a trial wave function proportional to $\exp \left(u_{+} \rho_{+}^{2}+u_{-} \rho_{-}^{2}\right)$ where

$$
2 u_{ \pm}=-1 / S+\left[1 / S^{2}+4 m \tilde{V}_{ \pm} / \hbar^{2} k^{2}\right]^{1 / 2} .
$$

The exponent becomes $\left(u_{+}+u_{-}\right)\left(\rho_{1}^{2}+\rho_{2}^{2}\right)+2\left(u_{+}-\right.$ $\left.u_{-}\right) \rho_{1} \rho_{2}$. Rapisarda and co-workers [19] used a correlation factor

$$
4 u_{ \pm}^{\prime}=-1 / S+\left[1 / S^{2}+8 m \tilde{V}_{ \pm} / \hbar^{2} k^{2}\right]^{1 / 2} .
$$

This can be interpreted as a different normalization factor $r=\sqrt{2}$. For the electron-electron system, in the limit that $d$ approaches zero so that $\tilde{V}_{a}=\tilde{V}_{e}$ and $\tilde{V}_{-}=0$, we get back the correct limit of correlation involving the particle-particle correlation. This is not true with the correlation factor in Eq. (6). We found numerically that Eq. (3) gives the lowest variational energy where possible. Equation (6) gives the highest energy. A similar generalization is used for the solid case. We have performed calculations with a sample of 30 electrons and 30 holes for different number of electron valleys $n_{v}(1,2,3,5)$ and doubly degenerate holes. The energies are then fitted with a quadratic polynomial in $1 / \sqrt{n_{v}}$ and extrapolated to provide for estimates of the energies for $n_{v}=6,12$. Our results are expressed in terms of the usual dimensionless density parameter $r_{s}$ defined by $\pi\left(r_{s} a_{B}\right)^{2}=1 / \sigma$. The energies for $n_{v}=6$ in units of Ry of different phases as a function of $r_{s}$ from fixed-node diffusion Monte Carlo simulations for three values of $d$ are shown in Fig. 6. At small $r_{s}$, we expect the kinetic energy term that is proportional to $1 / r_{s}^{2}$ to dominate. The intraplane potential energy that is proportional to $-1 / r_{s}$ comes in as $r_{s}$ increases. For large $r_{s}$ the energy per particle for the solid phase is of the order of half the exciton energies. This is consistent with results in Fig. 4. Our energy may be measured experimentally as it is equal to $\left(e^{2} \sigma / 2\right) /(C / A)$ where $C / A$ is the total capacitance $(C)$ per unit area $(A)$ of the system. The total capacitance contains contributions from the self-capacitances due to the e-e and h-h interactions and the mutual capacitance from the e-h interaction. Such type of capacitance measurements have recently been carried out by Ma and co-workers [32] on similar structures.

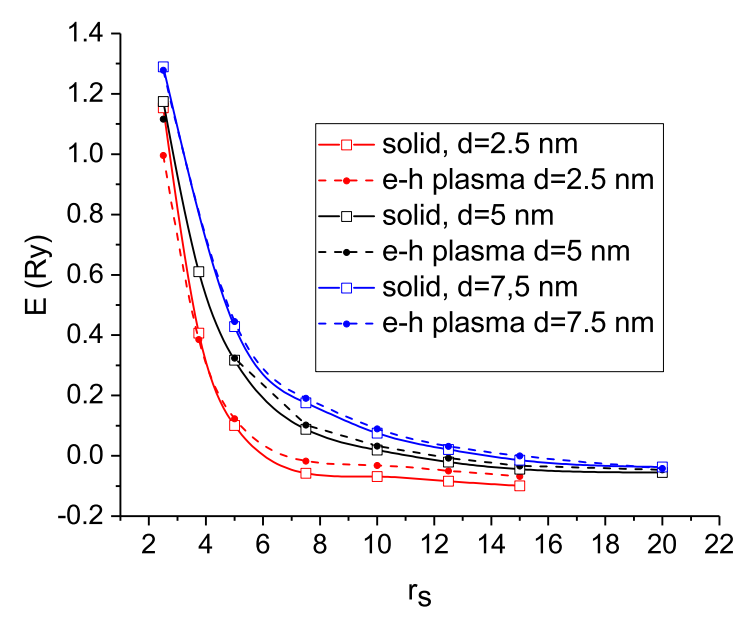

FIG. 6. The energies in units of $\mathrm{Ry}_{B N}$ of the solid (solid lines) and the e-h plasma (dashed lines) as a function of $r_{s}$ from fixed-node MC simulations for three values of $d_{B N}$ as is indicated by the inset in the figure.

From where the solid lines cross the dashed lines we obtain the phase boundary of the transition in Fig. 7. The physics involved in the transition is captured by an idea of Mott that as the density of the plasma is increased the screening length is reduced and it becomes more and more difficult to form an exciton. Thus the metallic plasma is more stable at high densities (small $r_{s}$ ). As the number of electron valleys is increased, the kinetic energy of the plasma is reduced, whereas the energy of the exciton is not much changed. The plasma has lower energy over a larger region in phase space. As the spacer thickness is decreased, the interlayer Coulomb interaction enhances the energy of the exciton and the coupling of density modulation $\left\langle\rho_{G}\right\rangle$ at reciprocal lattice vector $G$ that characterizes the solid phase. Thus the metallic region shrinks. The transition electron/hole density is in an experimentally accessible range between $4.1 \times 10^{12} \mathrm{~cm}^{-2}$ and $14.5 \times 10^{12} \mathrm{~cm}^{-2}$. This raises the possibility of exploiting the structure for logic device applications.

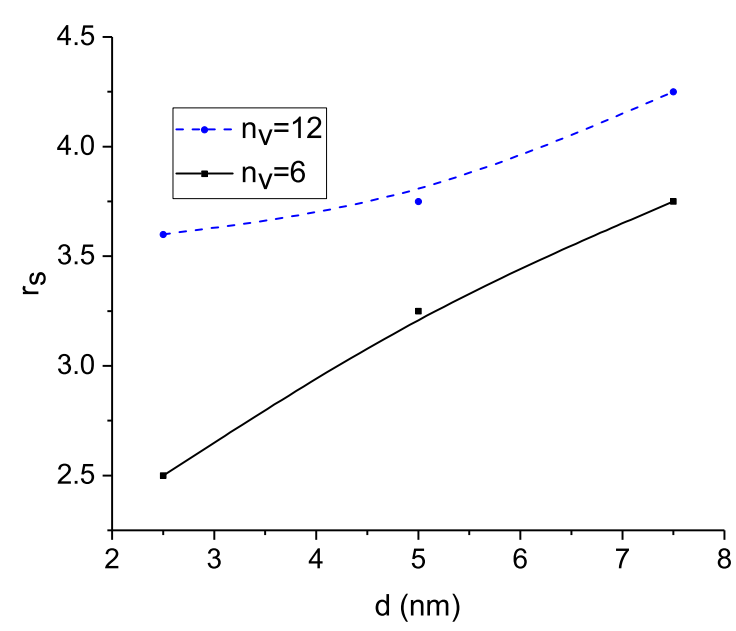

FIG. 7. The transition $r_{s}$ as a function of $d_{B N}$. 


\section{CONCLUSION}

In conclusion, we have investigated the metal-insulator transition between the electron-hole plasma and a newly proposed exciton solid for double-layer two-dimensional electron-hole systems in transition metal dichalcogenides stacked on opposite sides of thin layers of BN. We computed the energies of the two phases with fixed-node diffusion Monte Carlo simulation. The valley degeneracy of the bands lowers the energy of the e-h plasma by a significant amount. The screened interlayer and intralayer Coulomb interaction is calculated for typical realistic experimental structures by including the polarization charges at the interfaces of encapsulations and substrates. We found that the encapsulation can lower the exciton energy by an order of magnitude. For spacer thickness $d$ between 2.5 and $7.5 \mathrm{~nm}$, the transition electron/hole density is in an experimentally accessible range between $4.1 \times 10^{12} \mathrm{~cm}^{-2}$ and $14.5 \times 10^{12} \mathrm{~cm}^{-2}$. We have explored the effect of the potential from the charges on the boron and the nitrogen ions and found that it produced a less than $10 \%$ change in the energy difference between the two phases [33]. The phase boundary is not affected. Our results raise the possibility of exploiting this effect for logic device applications.

In this paper we focus on the case with equal number of electrons and holes. In the metallic phase, when the number of electrons is not equal to the number of holes, the state will remain metallic. In the insulating phase, we expect a small number of the excess electrons/holes to form a Wigner solid occupying the interstitial positions of the exciton solid [34], since the Wigner solid is stable at low densities. The system remains insulating. Eventually when the imbalance gets bigger the Wigner solid becomes unstable and the system becomes metallic. This metal insulator transition provides for another opportunity to explore possible device applications.

\section{ACKNOWLEDGMENTS}

N.W. acknowledges support from the National Key R\&D Program of China (2020YFA 0309600/0309602) and the Research Grants Council of Hong Kong (Project No. 16303720). B.T. is supported by TUBITAK under Grant No. 119N086 and Turkish Academy of Sciences (TUBA) under Grant No. AG21.
[1] X. Liu, K. Watanabe, T. Taniguchi, B. I. Halperin, and P. Kim, Nat. Phys. 13, 746 (2017).

[2] J. I. A. Li, T. Taniguchi, K. Watanabe, J. Hone, and C. R. Dean, Nat. Phys. 13, 751 (2017); C. Zhang and G. Jin, J. Phys.: Condens. Matter 25, 425604 (2013).

[3] J. I. A. Li, T. Taniguchi, K. Watanabe, J. Hone, A. Levchenko, and C. R. Dean, Phys. Rev. Lett. 117, 046802 (2016).

[4] K. Lee, J. Xue, D. C. Dillen, K. Watanabe, T. Taniguchi, and E. Tutuc, Phys. Rev. Lett. 117, 046803 (2016); S. Kim and E. Tutuc, Solid State Commun. 152, 1283 (2012).

[5] Z. Wang, D. A. Rhodes, K. Watanabe, T. Taniguchi, J. C. Hone, J. Shan, and K. F. Mak, Nature (London) 574, 76 (2019).

[6] S. T. Chui, N. Wang, and C. Y. Wan, Phys. Rev. B 102, 125420 (2020).

[7] A. Gamucci, D. Spirito, M. Carrega, B. Karmakar, A. Lombardo, M. Bruna, L. N. Pfeiffer, K. W. West, A. C. Ferrari, M. Polini, and V. Pellegrini, Nat. Commun. 5, 5824 (2014); M. Kellogg, J. P. Eisenstein, L. N. Pfeiffer, and K. W. West, Phys. Rev. Lett. 93, 036801 (2004); E. Tutuc, M. Shayegan, and D. A. Huse, ibid. 93, 036802 (2004); A. F. Croxall, K. Das Gupta, C. A. Nicoll, M. Thangaraj, H. E. Beere, I. Farrer, D. A. Ritchie, and M. Pepper, ibid. 101, 246801 (2008); J. A. Seamons, C. P. Morath, J. L. Reno, and M. P. Lilly, ibid. 102, 026804 (2009); J. P. Eisenstein, A. D. K. Finck, D. Nandi, L. N. Pfeiffer, and K. W. West, J. Phys: Conf. Ser. 456, 012009 (2013).

[8] W. F. Brinkman, T. M. Rice, P. W. Anderson, and S. T. Chui, Phys. Rev. Lett. 28, 961 (1972).

[9] M. Combescot and P. Noziéres, J. Phys. C: Solid State Phys. 5, 2369 (1972).

[10] V. M. Asnin, A. A. Rogachev, and N. I. Sablina, Sov. Phys.JETP Lett. 11, 99 (1970); C. Benoît à la Guillaume, M. Voos, and F. Salvan, Phys. Rev. B 5, 3079 (1972).

[11] J. P. Eisenstein and A. H. MacDonald, Nature (London) 432, 691 (2004); B. N. Narozhny and A. Levchenko, Rev. Mod. Phys. 88, 025003 (2016); B. Debnath, Y. Barlas, D.
Wickramaratne, M. R. Neupane, and R. K. Lake, Phys. Rev. B 96, 174504 (2017); L. Rademaker, J. van den Brink, J. Zaanen, and H. Hilgenkamp, ibid. 88, 235127 (2013).

[12] P. G. Silvestrov and P. Recher, Phys. Rev. B 95, 075438 (2017).

[13] The experimental data for the effective masses are higher than the theoretical ones. For $p$-type holes in $\mathrm{WSe}_{2}$ we have used the value of $0.8-0.9 m_{e}$; see S. Xu, J. Shen, G. Long, Z. Wu, Z.-q. Bao, C.-C. Liu, X. Xiao, T. Han, J. Lin, Y. Wu, H. Lu, J. Hou, L. An, Y. Wang, Y. Cai, K. M. Ho, Y. He, R. Lortz, F. Zhang et al., Phys. Rev. Lett. 118, 067702 (2017); However, an angle-resolved photoemission spectroscopy paper reports the effective mass of $\mathrm{WSe}_{2}$ holes to be $1.4 \pm 0.6 m_{e}$ at $K$ valley and $3.5 \pm 1.8 m_{0}$ at $\Gamma$ valley; see P.-C. Yeh, W. Jin, N. Zaki, D. Zhang, J. T. Liou, J. T. Sadowski, A. Al-Mahboob, J. I. Dadap, I. P. Herman, P. Sutter, and R. M. Osgood, Jr., Phys. Rev. B 91, 041407(R) (2015); M. V. Gustafsson, M. Yankowitz, C. Forsythe, D. Rhodes, K. Watanabe, T. Taniguchi, J. Hone, X. Zhu, and C. R. Dean reported in $K$ valley the relative effective masses for $\mathrm{WSe}_{2}$ of 0.45-0.55 (electrons) and 0.45-0.6 (holes). See Nat. Mater. 17, 411 (2018); We have used the electron effective mass of 0.5 . For $\mathrm{MoS}_{2}$ (n-type), the effective mass is about $0.55 m_{e}$; see J. Lin, T. Han, B. A. Piot, Z. Wu, S. Xu, G. Long, L. An, P. Cheung, P.-P. Zheng, P. Plochocka et al., Nano Lett. 19, 1736 (2019); The theoretical band structures in TMDCs are described in G.-B. Liu, W.-Y. Shan, Y. Yao, W. Yao, and D. Xiao, Phys. Rev. B 88, 085433 (2013); For some theoretical estimate of the masses, see A. Kormányos et al., 2D Mater. 2, 022001 (2015).

[14] M. M. Fogler, L. V. Butov, and K. S. Novoselov, Nat. Commun. 5, 4555 (2014).

[15] For parameters of bilayer graphene, see K. Zou, X. Hong, and J. Zhu, Phys. Rev. B 84, 085408 (2011).

[16] D. Ceperley, Phys. Rev. B 18, 3126 (1978).

[17] S. T. Chui and B. Tanatar, Phys. Rev. Lett. 74, 458 (1995). 
[18] R. Maezono, P. López Ríos, T. Ogawa, and R. J. Needs, Phys. Rev. Lett. 110, 216407 (2013).

[19] F. Rapisarda and G. Senatore, Aust. J. Phys. 49, 161 (1996); F. Rapisarda and S. Conti, Int. J. Mod. Phys. B 13, 479 (1999); S. De Palo, F. Rapisarda, and G. Senatore, Phys. Rev. Lett. 88, 206401 (2002).

[20] S. Narasimhan and T.-L. Ho, Phys. Rev. B 52, 12291 (1995).

[21] L. Swierkowski, D. Neilson, and J. Szymanski, Phys. Rev. Lett. 67, 240 (1991).

[22] A. Perali, D. Neilson, and A. R. Hamilton, Phys. Rev. Lett. 110, 146803 (2013).

[23] G. Goldoni, V. Schweigert, and F. M. Peeters, Surf. Sci. 361362, 163 (1996).

[24] M. Zarenia, D. Neilson, and F. M. Peeters, Sci. Rep. 7, 11510 (2017).

[25] D. A. Ruiz-Tijerina, M. Danovich, C. Yelgel, V. Zólyomi, and V. I. Fal'ko, Phys. Rev. B 98, 035411 (2018).

[26] J. D. Jackson, Classical Electrodynamics (Wiley, New York, 1962).
[27] L. V. Keldysh, Pis'ma Zh. Eksp. Teor. Fiz. 29, 716 (1979) [JETP Lett. 29, 658 (1979)].

[28] A. Chernikov, T. C. Berkelbach, H. M. Hill, A. Rigosi, Y. Li, O. B. Aslan, D. R. Reichman, M. S. Hybertsen, and T. F. Heinz, Phys. Rev. Lett. 113, 076802 (2014).

[29] A. Laturia, M. L. Van de Put, and W. G. Vandenberghe, 2D Mater. Appl. 2, 6 (2018).

[30] For some examples, see L. Bonsall and A. Maraduddin, Phys. Rev. B 15, 1959 (1977); the first terms on the right-hand sides of Eqs. (2-15)-(2-21).

[31] B. Tanatar and D. M. Ceperley, Phys. Rev. B 39, 5005 (1989), and references therein.

[32] L. Ma, P. X. Nguyen, Z. Wang, Y. Zeng, K. Watanabe, T. Taniguchi, A. H. MacDonald, K. F. Mak, and J. Shan, Nature (London) 598, 585 (2021).

[33] S. T. Chui (unpublished).

[34] S. T. Chui and K. Esfarjani, Phys. Rev. B 44, 11498 (1991); 45, 11441 (1992); N. Wang and S. T. Chui (unpublished). 